\title{
Coupling of erbium-implanted silicon to a superconducting resonator
}

Mark A. Hughes, ${ }^{* 1}$ Naitik A. Panjwani, ${ }^{2,3}$ Matias Urdampilleta,,${ }^{2,4}$ Nafsika Theodoropoulou, ${ }^{1}$ Ilana Wisby, ${ }^{5,6,7}$ Kevin P. Homewood, ${ }^{8,9}$ Ben Murdin, ${ }^{8}$ Tobias Lindström ${ }^{5}$ and J. David Carey ${ }^{8,10}$

${ }^{1}$ Materials and Physics Research Group, School of Science, Engineering and Environment, University of Salford, Salford, M5 4WT, UK

${ }^{2}$ University College London, London Centre for Nanotechnology, Gower Place, WC1E 6BT, London, UK

${ }^{3}$ Berlin Joint EPR Lab, Fachbereich Physik, Freie Universität Berlin, D-14195 Berlin, Germany ${ }^{4}$ Institut Néel-CNRS-UJF-INPG, UPR2940 25 rue des Martyrs BP 166, 38042 Grenoble cedex 9, France

${ }^{5}$ National Physical Laboratory, Hampton Road, Teddington TW11 OLW, UK

${ }^{6}$ Royal Holloway, University of London, Egham TW20 0EX, UK

${ }^{7}$ Oxford Quantum Circuits Ltd. King Charles House 2nd Floor, Park End Street, Oxford, Oxfordshire, OX1 1JD, UK

${ }^{8}$ Advanced Technology Institute, Faculty of Engineering and Physical Sciences, University of Surrey, Guildford, GU2 7XH, UK

${ }^{9}$ School of Materials Science and Engineering, Hubei University, Wuhan, 430062, Peoples Republic of China

${ }^{10}$ Department of Electrical and Electronic Engineering, University of Surrey, Guildford, GU2 7XH, UK

"Email: m.a.hughes@salford.ac.uk 


\begin{abstract}
Erbium implanted silicon is promising for both photonic and quantum technology platforms, since it possesses both telecommunications and integrated circuit processing compatibility. However, several different Er centres are generated during the implantation and annealing process, the presence of which could hinder the development of these applications. When Si is co-implanted with $10^{17} \mathrm{~cm}^{-3} \mathrm{Er}$ and $10^{20} \mathrm{~cm}^{-3} \mathrm{O}$ ions, and the appropriate annealing process is used, one of these centres, which is present at higher Er concentrations, can be eliminated. Characterisation of samples with Er concentrations $<10^{17} \mathrm{~cm}^{-3} \mathrm{Er}$ is limited by the sensitivity of standard electron paramagnetic resonance (EPR) instruments. The collective coupling strength between a superconducting (SC) NbN lumpedelement resonator and a $10^{17} \mathrm{~cm}^{-3} \mathrm{Er}$ implanted Si sample at $20 \mathrm{mK}$ was measured to be $\sim 1 \mathrm{MHz}$, which provides a basis for the characterisation of low concentration Er implanted Si and for future networks of hybrid quantum systems that exchange quantum information over the telecommunication network. Out of six known Er-related EPR centres, only one trigonal centre coupled to the SC resonator.
\end{abstract}




\section{INTRODUCTION}

When Er is co-implanted with $\mathrm{O}$ into $\mathrm{Si}$, a variety of EPR and photoluminescence (PL) active centres can be observed. The PL active centres have been proposed as a platform for developing all-silicon photonic technology solutions that would eliminate the undesirable need for discrete direct bandgap semiconductor devices to interface with the fibre optic telecommunications network [1]. The EPR active centres have been proposed as a platform for developing quantum technologies (QTs) since using Er implanted Si links the decoupling from the environment of the partially filled $4 f$-shell inherent to all rare earths, with the integrated circuit fabrication pedigree of silicon $[2,3]$. We have recently reported an electron spin coherence time from Er implanted $\mathrm{Si}$, with an Er concentration of $3 \times 10^{17} \mathrm{~cm}^{-3}$, of $7.5 \mu$ s at $5 \mathrm{~K}$ [4], which compares to $1.6 \mu \mathrm{s}$ at $1.9 \mathrm{~K}$ for $\sim 2 \times 10^{18} \mathrm{~cm}^{-3} \mathrm{Er} \mathrm{Y}_{2} \mathrm{SiO}_{5}[5]$ and $\sim 5 \mu$ s at $5 \mathrm{~K}$ for $\sim 10^{16} \mathrm{~cm}^{-3} \mathrm{Er}_{\mathrm{CaWO}}[6]$. This is promising given the difficulty in repairing defects after implantation that could lead to decoherence. To date, one of the main issues in the development of the photonic and quantum technology applications of Er implanted Si has been the difficulty in characterising, identifying and controlling the large number of EPR and PL active centres that are generated; a solution to this problem is offered through coupling the Er spin ensemble to a superconducting (SC) resonator, which can provide sensitivity enhancements over traditional EPR techniques. For example, by exploiting $\mathrm{SC}$ resonator coupling, the EPR signal from $\mathrm{Er}: \mathrm{Y}_{2} \mathrm{SiO}_{5}$ can be detected with $\sim 1 \mathrm{fW}$ of microwave power [7] and the sensitivity for Bi spins in $\mathrm{Si}$ is 65 spins $/ \sqrt{ } \mathrm{Hz}$ [8].

As well as offering advantages for the EPR characterisation of a spin ensemble, the coupling of an SC resonator to a spin ensemble can itself constitute a hybrid quantum system with many advantages for quantum information processing over either individual system. If the interactions of a superconducting resonator coupled to a spin ensemble are mediated with one or a few superconducting qubits, a system possessing both the long coherence times of the spin ensemble and the fast gating of the superconducting qubits can be formed, in which case the hybrid quantum system can behave as a quantum Turing machine with the spin ensemble forming a microwave quantum memory and the SC qubits performing the gate operations $[9,10]$. The use of rare earths as a spin ensemble is beneficial because they generally possess large $g$ factors as the orbital angular momentum is not quenched, which is advantageous for strong coupling [11], and long coherence times, which is 
advantageous for quantum memory [2]. The successful storage and on-demand retrieval of 16 weak coherent microwave pulses from $\mathrm{Er}_{2} \mathrm{Y}_{2} \mathrm{SiO}_{5}$ has been demonstrated [12], and a single ensemble could be used to store many qubits by using holographic encoding [13]. In addition, ensembles of rare earth ions coupled to SC resonators have been proposed for microwave-to-optical transducers for quantum networks $[11,14]$. The coherent conversion of single microwave to telecoms wavelength photons would allow applications such as optical fibre links between separate quantum processors and the deterministic entanglement of telecoms wavelength photons [15]. We have previously demonstrated that under $1.5 \mu \mathrm{m}$ illumination an EPR resonance from a PL active Er centre with orthorhombic symmetry in Er implanted Si can be observed which has an intensity three orders of magnitude higher than unilluminated EPR resonances [2]. This shows that the microwave and optical transitions required for various proposed microwave-to-optical transducer schemes involving rare earths[16,17] are present in a single Er centre in Er implanted Si. Other features that make Er implanted Si attractive for quantum information processing include the demonstration of deterministic single-ion implantation of rare earth ions with a placement precision of $34 \mathrm{~nm}$ [18].

Optical and magnetic characterisation of Er implanted Si presents a significant challenge in detecting a PL or EPR signal because of the relatively low number of Er centres compared to bulk, the low implant yield for any particular centre (the yield for all optically active Er centres varies between 1 and $10 \%$ for different studies [1]), and the indirect band gap of silicon. As such, EPR and PL characterisation is typically carried out with a high Er concentration, typcially $10^{19} \mathrm{~cm}^{-3}[1,19]$. Here we report EPR and PL from a sample with as low as $10^{17} \mathrm{~cm}^{-3} \mathrm{Er}$, and the first coupling between Er implanted in $\mathrm{Si}$ and a superconducting resonator, which could have applications in further characterisation and identification of the centres of Er implanted Si to enable quantum and photonic technology applications and in the development microwave quantum memory and microwave-tooptical transducers. 


\section{EXPERIMENTAL}

Two samples, with Er concentrations of $10^{17} \mathrm{~cm}^{-3}$ and $10^{19} \mathrm{~cm}^{-3}$, both with $\mathrm{O}$ concentrations of $10^{20}$ $\mathrm{cm}^{-3}$, were prepared by implanting Er and O ions into $<100>$ oriented $8000 \pm 500 \Omega \mathrm{cm} \mathrm{Si}$ wafer. Samples were then annealed at $620^{\circ} \mathrm{C}$ for $180 \mathrm{~min}$ anneal to recrystallize the amorphized region, then annealed at $850^{\circ} \mathrm{C}$ for $30 \mathrm{~s}$ to activate the Er; it was previously found that annealing at $850^{\circ} \mathrm{C}$ significantly increased the EPR signal strength [2]. For all samples, $\mathrm{O}$ and Er ions were implanted at a range of energies to give a flat concentration profile down to a depth of around $1.5 \mu \mathrm{m}$, see supplementary Figure S1 [20]. Isotope specific implantation was used so that only the zero nuclear spin ${ }^{166} \mathrm{Er}$ was implanted.

EPR measurements were performed in a Bruker E580 EPR spectrometer, with the magnetic field, $\mathrm{B}_{0}$, parallel to the [001] direction of the wafer with an uncertainty of $\pm 5^{\circ}$. All EPR measurements were made at $10 \mathrm{~K}$, with a microwave frequency of $9.61 \mathrm{GHz}$, and a modulation width of $2 \mathrm{G}$. The microwave power used was $0.5 \mathrm{~mW}$ and $2.5 \mathrm{~mW}$ for the $10^{17} \mathrm{~cm}^{-3} \mathrm{Er}$ and $10^{19} \mathrm{~cm}^{-3} \mathrm{Er}$ samples, respectively. PL spectra were obtained by cooling the sample in a cold finger $\mathrm{LN}_{2}$ cryostat at $65 \mathrm{~K}$. Excitation was by a $462 \mathrm{~nm} 10 \mathrm{~mW}$ laser diode and the generated fluorescence was dispersed in a Bentham TMc300 monochromator, with a resolution of $1 \mathrm{~nm}$, and detected with an IR photo multiplier tube coupled with standard phase sensitive detection.

SC resonator coupling measurements were performed in a dilution refrigerator, fitted with a vector magnet, at $20 \mathrm{mK}$. A superconducting lumped element micro-resonator was fabricated by sputtering $200 \mathrm{~nm}$ of $\mathrm{NbN}$, patterned by standard e-beam lithography, onto an R-cut sapphire substrate. The $10^{17}$ $\mathrm{cm}^{-3}$ Er sample was "flip-chipped" on top of the micro-resonator and pressed down using $\mathrm{Cu}-\mathrm{Be}$ springs. The micro-resonator was placed in a magnetic field that was stepped from zero to $930 \mathrm{G}$. The power in the resonator was $\sim 3 \mathrm{pW}$. At each magnetic field the microwave transmission coefficient, $\mathrm{S}_{21}$, was measured using a vector network analyser (VNA). This was repeated for magnetic field orientations between $0^{\circ}$ and $160^{\circ}$ in steps of $5^{\circ}$, with $0^{\circ}$ corresponding to $\mathrm{B}_{0}$ parallel to the face of the resonator and sample. The magnetic field was rotated around the [110] crystal axis of the sample. Numerical fitting of the $S_{21}$ response of the micro-resonator was used to extract the total measured loss tangent $\tan \delta_{\text {tot }}=1 / \mathrm{Q}_{\mathrm{tot}}$, where $\mathrm{Q}_{\mathrm{tot}}$ is the total measured Q factor and $\tan \delta_{\mathrm{tot}}=\tan \delta_{\mathrm{c}}+\tan \delta_{\text {diel }}+$ 
$\tan \delta_{\mathrm{B}}+\tan \delta_{\text {ions }}$, are the loss tangents due to coupling to the transmission line, dielectric losses, the external magnetic field and the Er ions, respectively. Numerical fitting was used to extract $\tan \delta_{\text {ions. }}$ A discussion of the loss mechanisms is given in the supplementary information [20].

\section{RESULTS AND DISCUSSION}

\section{A. EPR measurements}

When implanted into Si, Er exists in its usual 3+ oxidation state [1]. Oxygen was co-implanted to a concentration of $10^{20} \mathrm{~cm}^{-3}$ for both samples and is required to generate narrow Er-related EPR [21] and PL [22] lines by the creation of various O coordinated Er centres. PL lines from a Si coordinated Er centre with cubic symmetry, Er-C, can be observed without O co-implantation [22]. PL lines from an $\mathrm{O}$ coordinated Er centre with orthorhombic $\mathrm{C}_{2 \mathrm{v}}$ symmetry, and an unidentified $\mathrm{O}$ coordinated $\mathrm{Er}$ centre, referred to as Er-O1R and PL-U, respectively, have been reported [2]. Previous measurements of the angular dependence of the Er-related EPR lines in Er implanted Si have identified a number of different $\mathrm{O}$ coordinated Er EPR centres: three monoclinic $\mathrm{C}_{1 \mathrm{~h}}$ centres labelled $\mathrm{OEr}-1, \mathrm{OEr}-1$ ' and OEr-3, and three trigonal $C_{3 v}$ centres labelled OEr-2, OEr-2'and OEr-4 [19,21,23,24]. An unidentified EPR centre, EPR-U, with resonances $\sim 1700 \mathrm{G}$, using the same $\mathrm{B}_{0}$ orientation as us, has been reported [21]. Zeeman measurements of molecular-beam epitaxy (MBE) grown Er doped Si have identified an orthorhombic O coordinated Er centre [25], which is not EPR active and we have argued is the same centre as the Er-O1R we have previously identified [2]. A summary of all these centres, including their $g$-factors, are given in Table I. The isotropic $g$-factor for the cubic centre was calculated from crystal field analysis of the PL measurements [26]. 
Table I Summary of the known EPR and PL active centres in Er implanted Si

\begin{tabular}{|l|l|c|c|c|c|c|}
\hline Centre & Symmetry & $\begin{array}{c}\text { Local } \\
\text { Coordination }\end{array}$ & $\mathbf{g}_{\mathbf{x}}$ & $\mathbf{g}_{\mathbf{y}}$ & $\mathbf{g}_{\mathbf{z}}$ & Ref. \\
\hline \multicolumn{7}{|c|}{ EPR active centres } \\
\hline OEr-1 & Monoclinic $\mathrm{C}_{1 \mathrm{~h}}$ & $\mathrm{O}$ & 0.8 & 5.45 & 12.6 & {$[21]$} \\
\hline OEr-1' & Monoclinic $\mathrm{C}_{1 \mathrm{~h}}$ & $\mathrm{O}$ & 0.8 & 5.45 & 12.55 & {$[21]$} \\
\hline OEr-3 & Monoclinic $\mathrm{C}_{1 \mathrm{~h}}$ & $\mathrm{O}$ & 1.09 & 5.05 & 12.78 & {$[21]$} \\
\hline OEr-4 & Trigonal $\mathrm{C}_{3 \mathrm{v}}$ & $\mathrm{O}$ & 2.0 & 6.23 & 6.23 & {$[21]$} \\
\hline OEr-2 & Trigonal $\mathrm{C}_{3 \mathrm{v}}$ & $\mathrm{O}$ & 0.45 & 3.46 & 3.22 & {$[21]$} \\
\hline OEr-2' & Trigonal $\mathrm{C}_{3 \mathrm{v}}$ & $\mathrm{O}$ & 0.69 & 3.24 & 3.24 & {$[21]$} \\
\hline EPR-U & - & $\mathrm{O}$ & - & - & - & {$[21]$} \\
\hline & & PL active centres & & & \\
\hline Er-O1R & Orthorhombic $\mathrm{C}_{2 \mathrm{v}}$ & $\mathrm{O}$ & $\sim 0$ & $\sim 0$ & 18.4 & {$[2,25]$} \\
\hline Er-C & Cubic $\mathrm{T}_{\mathrm{d}}$ & $\mathrm{Si}$ & 6.8 & - & - & {$[2,21,26]$} \\
\hline PL-U & - & $\mathrm{O}$ & - & - & - & {$[2]$} \\
\hline
\end{tabular}

Figure 1 shows the EPR spectra of the $10^{17} \mathrm{~cm}^{-3}$ Er and $10^{19} \mathrm{~cm}^{-3}$ Er samples. By comparison to the angular dependencies of the previously reported EPR centres in Table I we can assign a unique centre to all of the observed resonances. Note that a small angular deviation in the [1 $\overline{1} 0]$ axis can cause significant shifting of the EPR resonances. The most intense resonances for both the $10^{17} \mathrm{~cm}^{-3} \mathrm{Er}$ and $10^{19} \mathrm{~cm}^{-3}$ Er samples at $\sim 925 \mathrm{G}$, attributed to the OEr-1 and OEr-3 monoclinic centres all have widths of $\sim 5 \mathrm{G}$, whereas the resonances for both samples at $\sim 2200 \mathrm{G}$, attributed to the OEr-2' trigonal centres all have widths of $\sim 20 \mathrm{G}$. The independence of linewidth over such a large concentration range indicates that clustering does not affect the spin linewidth and therefore both centres are homogenously distributed. In $\mathrm{Er}: \mathrm{Y}_{2} \mathrm{SiO}_{5}$ there are two Er centres with different $g$-factors; when coupled to a SC resonator it was found that the spin linewidth of the centre with a smaller $g$-factor was greater than the centre with a large $g$-factor; this was attributed to inhomogeneities in the DC magnetic field [27]. However, transitions with a larger $g$-factor are more susceptible to inhomogeneities in the magnetic field, which also broadens the inhomogeneous spin linewidth. The 
balance of these effects depends on alignment [27], which could explain the difference in EPR linewidth between the monoclinic and trigonal Er centres that we observed.

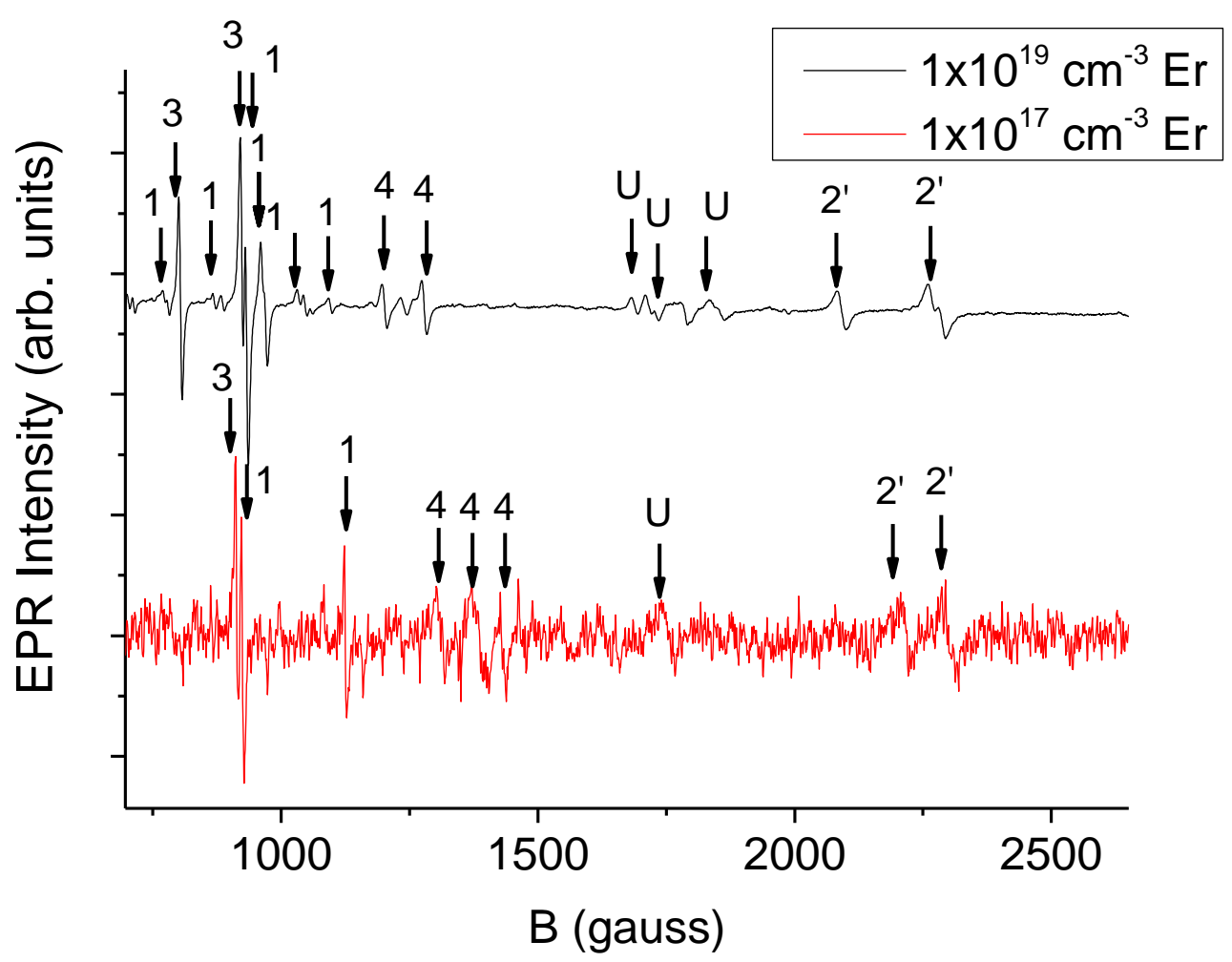

FIG 1 EPR spectra of $10^{17} \mathrm{~cm}^{-3} \mathrm{Er}$ and $10^{19} \mathrm{~cm}^{-3} \mathrm{Er}$ implanted Si. Resonances are assigned to the EPR centres in Table I, OEr-1, OEr-2' etc, as 1,2' etc, and U is the unidentified EPR centre. The microwave frequency was $9.61 \mathrm{GHz}$ and the magnetic field was parallel to the [001] direction of the wafer.

\section{B. Photoluminescence measurements}

Figure 2 shows the PL spectra of the $10^{17} \mathrm{~cm}^{-3} \mathrm{Er}$ and $10^{19} \mathrm{~cm}^{-3} \mathrm{Er}$ samples. In the $10^{19} \mathrm{~cm}^{-3} \mathrm{Er}$ spectrum we can identify peaks from the Er-C, the Er-O1R orthorhombic centre and PL-U unidentified centres [2] as indicated. The most intense peak at $\sim 6510 \mathrm{~cm}^{-1}$ can be attributed to both the Er-C and Er-O1R centres [2]. In the $10^{17} \mathrm{~cm}^{-3} \mathrm{Er}$ spectrum no peaks unique to the Er-C centre can be identified, indicating that the Er-C centre is not present in the $10^{17} \mathrm{~cm}^{-3} \mathrm{Er}$ sample. This indicates that the 1000:1 O:Er ratio in the $10^{17} \mathrm{~cm}^{-3}$ Er sample is effective in eliminating the Er-C Si coordinated centre because of the relative abundance of $\mathrm{O}$. Additional peaks at 6450 and $6495 \mathrm{~cm}^{-1}$ not observed 
in the $10^{19} \mathrm{~cm}^{-3} \mathrm{Er}$ spectrum can be identified, it cannot be determined at this stage if these originate from the same unidentified centre which gives peaks at $>6510 \mathrm{~cm}^{-1}$.

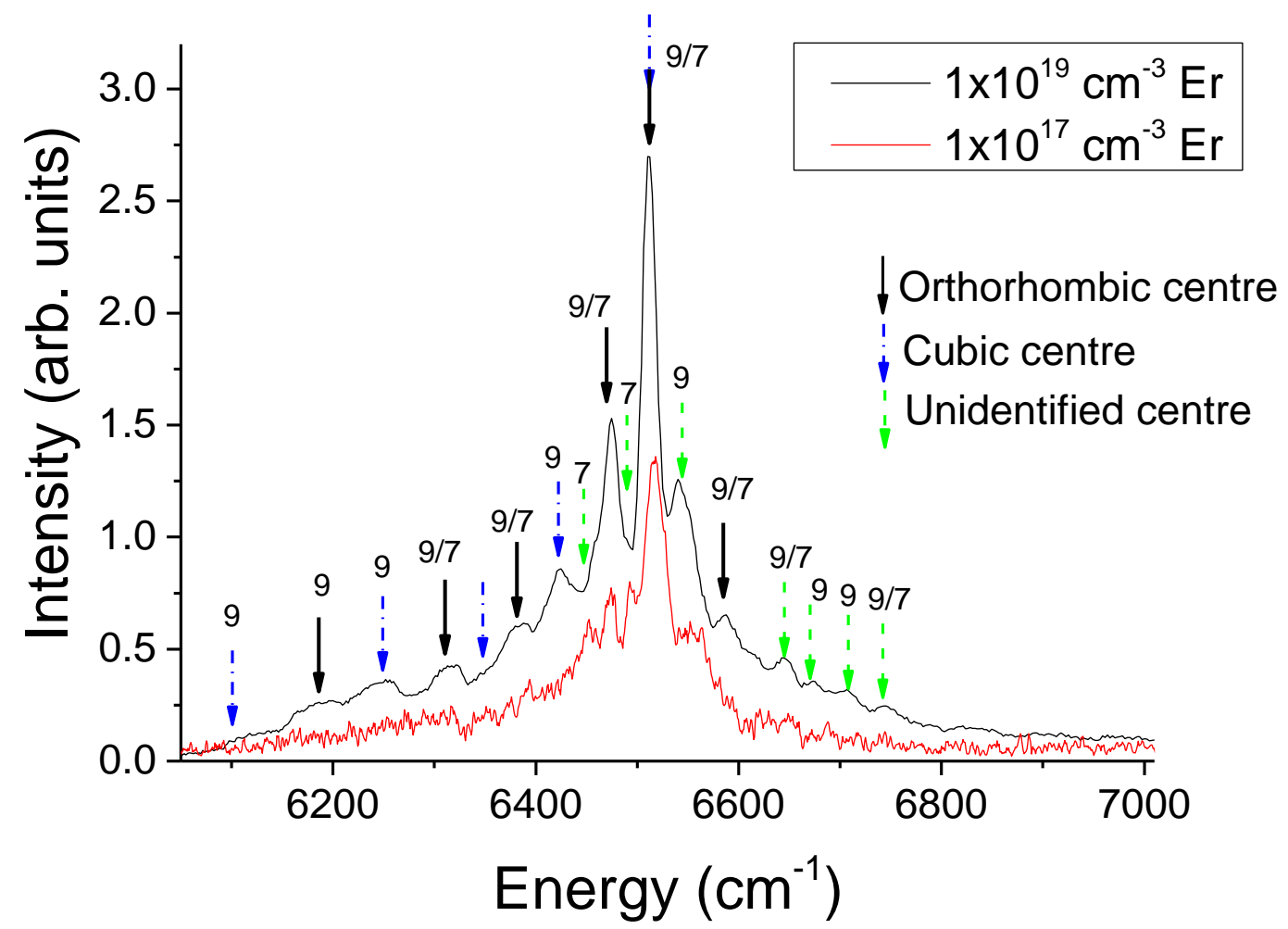

FIG 2 PL spectra of $10^{17} \mathrm{~cm}^{-3} \mathrm{Er}$ and $10^{19} \mathrm{~cm}^{-3} \mathrm{Er}$ implanted Si at $65 \mathrm{~K}$. PL peaks are assigned to the orthorhombic Er-O1R, cubic Er-C and unknow (PL-U) PL centres in Table I with arrows. The arrows are marked with 9,7 or 9/7 if the peaks can be identified in the $10^{19} \mathrm{~cm}^{-3} \mathrm{Er}$ spectrum, $10^{17} \mathrm{~cm}^{-3} \mathrm{Er}$ spectrum or both spectra, respectively.

The PL linewidths are broadly similar for the $10^{17} \mathrm{~cm}^{-3} \mathrm{Er}$ and $10^{19} \mathrm{~cm}^{-3} \mathrm{Er}$ spectra: $\sim 20 \mathrm{~cm}^{-1}$ for the identifiable peaks. There are some small but significant shifts in the peak positions attributed to the orthorhombic centre of the $10^{17} \mathrm{~cm}^{-3}$ Er spectrum compared to the $10^{19} \mathrm{~cm}^{-3}$ Er spectrum, particularly the main peak at $\sim 6510 \mathrm{~cm}^{-1}$ and the highest energy orthorhombic peak at $\sim 6570 \mathrm{~cm}^{-1}$, which could represent a subtle change in the structure of this centre.

Crystal field analysis of rare earth spectra can be used to find crystal field parameters which measure the interaction between the $f$-shell electrons of rare earths and their surrounding crystalline 
environment. Each possible symmetry of the rare earth's environment has its own set of CFPs. The Hamiltonian $(H)$ of a rare earth in a magnetic field can be described as

$$
H=H_{F}+H_{C F}+H_{Z e}
$$

$H_{F}$ accounts for the interactions that occur in a free rare earth ion and is responsible for the energy of the J manifolds. Each rare earth has its own set of $H_{F}$ parameters, which vary slightly between crystal hosts; those given by Carnall et al. for $\mathrm{Er}_{\mathrm{LaF}} \mathrm{La}_{3}$ were used here [28]. $H_{C F}$ represents the perturbation generated by the ligands field surrounding a rare earth ion. The multipole expansion of $H_{C F}$ is the linear combination of a set of spherical tensors, $C_{q}^{(k)}$, and a corresponding set of structural factors, $B_{q}^{k}$, which are the crystal field parameters.

$$
H_{C F}=\sum_{k, q} B_{q}^{k} C_{q}^{(k)}
$$

A description of the $C_{q}^{(k)}$ spherical tensor operator matrix can be found elsewhere [3]. Diagonalizing $H_{C F}$ gives the crystal field energy levels of the $J$ manifold. To find the CFPs we fitted CFPs that minimise the sum of squares difference between the calculated and experimental energy levels using the Levenberg-Marquardt algorithm. The Zeeman interaction, $H_{Z e}$, is given by

$$
H_{Z e}=g_{J} \mu_{B} J \cdot \boldsymbol{H}
$$

where $g_{J}$ is the Landé factor, $\mu_{B}$ is the Bohr magneton, $\boldsymbol{J}$ is the angular momentum operator, and $\boldsymbol{H}$ is the magnetic field strength [29]. $H_{Z e}$ was not considered during the fitting procedure. Once the $\mathrm{Er}^{3+}$ CFPs have been determined, the $g$-tensor for any crystal field doublet can be calculated from their eigenvectors, $|+\rangle$ and $|-\rangle$. The diagonal components of the $g$-tensor, $g_{\mathrm{x}}, g_{\mathrm{y}}, g_{\mathrm{z}}$, can then be calculated from the first order perturbation expressions [29].

$$
g_{x}=2 g_{J}\left\langle+\left|J_{x}\right|-\right\rangle, g_{y}=2 g_{J}\left\langle+\left|J_{y}\right|-\right\rangle, g_{z}=2 g_{J}\left\langle+\left|J_{z}\right|+\right\rangle
$$

where $\boldsymbol{J}_{x}, \boldsymbol{J}_{\boldsymbol{y}}, \boldsymbol{J}_{z}$ are the vector components of $\boldsymbol{J}$ such that $\boldsymbol{J}^{\mathbf{2}}=\boldsymbol{J}_{\boldsymbol{x}}^{\mathbf{2}}+\boldsymbol{J}_{\boldsymbol{y}}^{\mathbf{2}}+\boldsymbol{J}_{\boldsymbol{z}}^{\mathbf{2}}$. 
We fitted the orthorhombic $\mathrm{C}_{2 \mathrm{v}}$ set of CFPs to the Er-O1R centre PL lines in Fig. 2; the PL lines $<6200 \mathrm{~cm}^{-1}$ could not be resolved in the $10^{17} \mathrm{~cm}^{-3} \mathrm{Er}$ spectrum, so we used those from the $10^{19}$ $\mathrm{cm}^{-3}$ Er spectrum. We also used the hot line previously identified for this centre [22] to give the required number of crystal field levels to fit the nine orthorhombic $\mathrm{C}_{2 \mathrm{v}}$ CFPs, which are given in Table II.

Table II Fitted orthorhombic $\mathrm{C}_{2 \mathrm{v}}$ crystal field parameters for $10^{19} \mathrm{~cm}^{-3} \mathrm{Er}$ and $10^{17} \mathrm{~cm}^{-3} \mathrm{Er}$ PL.

\begin{tabular}{|l|c|c|c|c|c|c|c|c|c|}
\hline & \multicolumn{7}{|c|}{$\mathbf{C}_{2 \mathrm{v}}$ crystal field parameters $\left(\mathbf{c m}^{-\mathbf{1}}\right)$} \\
\hline $\begin{array}{l}\text { Er conc. } \\
\left(\mathbf{c m}^{-3}\right)\end{array}$ & $\boldsymbol{B}_{\mathbf{0}}^{\mathbf{2}}$ & $\boldsymbol{B}_{\mathbf{0}}^{\mathbf{4}}$ & $\boldsymbol{B}_{\mathbf{0}}^{\mathbf{6}}$ & $\boldsymbol{B}_{\mathbf{2}}^{\mathbf{2}}$ & $\boldsymbol{B}_{\mathbf{2}}^{\mathbf{4}}$ & $\boldsymbol{B}_{\mathbf{4}}^{\mathbf{4}}$ & $\boldsymbol{B}_{\mathbf{2}}^{\mathbf{6}}$ & $\boldsymbol{B}_{\mathbf{4}}^{\mathbf{6}}$ & $\boldsymbol{B}_{\mathbf{6}}^{\mathbf{6}}$ \\
\hline $\mathbf{1 0}^{\mathbf{1 9}}$ & $-72 \pm 3$ & $-2085 \pm 36$ & $3 \pm 7$ & $159 \pm 6$ & $-208 \pm 21$ & $262 \pm 11$ & $-260 \pm 7$ & $-109 \pm 4$ & $-30 \pm 10$ \\
\hline $\mathbf{1 0}^{\mathbf{1 7}}$ & $-58 \pm 10$ & $-1879 \pm 49$ & $18 \pm 8$ & $569 \pm 59$ & $-258 \pm 38$ & $214 \pm 24$ & $-284 \pm 29$ & $40 \pm 31$ & $4 \pm 26$ \\
\hline
\end{tabular}

To interpret the CFPs, recognising how the orthorhombic set of CFPs arises from progressive distortions of higher symmetry groups can be helpful. Cubic symmetry is described by four CFPs with constraints: $B_{4}^{4}=5 B_{0}^{4}$ and $B_{4}^{6}=-21 B_{0}^{6}$ [30]. With a tetragonal distortion to a cubic field, the $B_{0}^{2}$ parameter appears, so it belongs only to the tetragonal component of the crystal field [31], and is therefore dependent on the degree of axial asymmetry. An orthorhombic distortion to a purely tetragonal field is described by addition of the $B_{2}^{2}, B_{2}^{4}, B_{2}^{6}$ and $B_{6}^{6}$ parameters. The calculated CFPs for the $10^{19} \mathrm{~cm}^{-3}$ Er spectrum are almost identical to what we have previously determined for $10^{19} \mathrm{~cm}^{-3}$ Er [2]; however, there are some important differences in the $10^{17} \mathrm{~cm}^{-3}$ Er CFPs. $B_{0}^{2}$ is smaller indicating there is less tetragonal distortion, whereas the magnitude of the four CFPs representing an orthorhombic distortion are all greater, except for $B_{6}^{6}$, indicating a greater degree of orthorhombic distortion. Using the eigenvectors from our CFP fitting and Eq. 4 we calculated $g$ - factors for $10^{17} \mathrm{~cm}$ ${ }^{3}$ and $10^{19} \mathrm{~cm}^{-3} \operatorname{Er}$ as $g_{x} \approx 0, g_{y} \approx 0, g_{z}=17.6$ and $g_{x} \approx 0, g_{y} \approx 0, g_{z}=17.9$, respectively, showing that any change in the structure of the Er-O1R at $10^{17} \mathrm{~cm}^{-3}$ Er has an insignificant effect on the $g$-factor. 


\section{Superconducting resonator coupling}

The implanted face of the $10^{17} \mathrm{Er} \mathrm{cm}^{-3}$ sample was placed in contact with the superconducting $\mathrm{NbN}$ lumped-element micro-resonator on $\mathrm{R}$-cut $\mathrm{Al}_{2} \mathrm{O}_{3}$ shown in Fig. 3a, which had a centre frequency $\omega_{\mathrm{r}} / 2 \pi=3.04 \mathrm{GHz}$, see experimental section. Figure $3 \mathrm{~b}$ shows the loss tangents due to coupling to $\mathrm{Er}$ ions ( $\left.\tan \delta_{\text {ions }}\right)$ as function of $\mathrm{B}_{0}$ and orientation. There is a single narrow resonance, with a FWHM of $50 \pm 10 \mathrm{G}$, that varies smoothly between 740 and $870 \mathrm{G}$ depending on the $\mathrm{B}_{0}$ orientation. There is also a very broad resonance centred at $500 \mathrm{G}$ and at $\mathrm{B}_{0} / /[001]\left(0^{\circ}\right.$ orientation), the resonance shifts to 600 $\mathrm{G}$ at $50^{\circ} \mathrm{B}_{0}$ orientation; we simulated the angular dependence of the six EPR centres (three trigonal, three monoclinic) previously identified Er and O implanted Si system [21], see Table I, but found no correspondence with this broad resonance. The narrow resonance had a correspondence with the trigonal OEr-2' centre identified in ref. [21] with $\mathrm{g}_{\|}=0.69$ and $\mathrm{g} \perp=3.24$, which is shown in the simulation in Fig. 3c. The two other resonances are also visible in this $\mathrm{B}_{0}$ range but are significantly weaker, which explains why only one resonance is observed in the micro-resonator measurement. A higher $\mathrm{B}_{0}$ range shows the positions of all three expected EPR resonances with trigonal symmetry in the simulation in Fig. 3d. Only one previously identified OEr-2' trigonal centre is evident in the micro-resonator measurements at $20 \mathrm{mK}$; however, standard EPR measurements of the $10^{17} \mathrm{Er} \mathrm{cm}^{-3}$ at $10 \mathrm{~K}$ showed the presence of the OEr-1 monoclinic centre. This indicates that the spin-lattice relaxation time, $\mathrm{T}_{1}$, of the $\mathrm{OEr}-1$ centre is long enough at $20 \mathrm{mK}$ to cause saturation of the coupling signal, but not for the OEr-2' centre. 
(a)

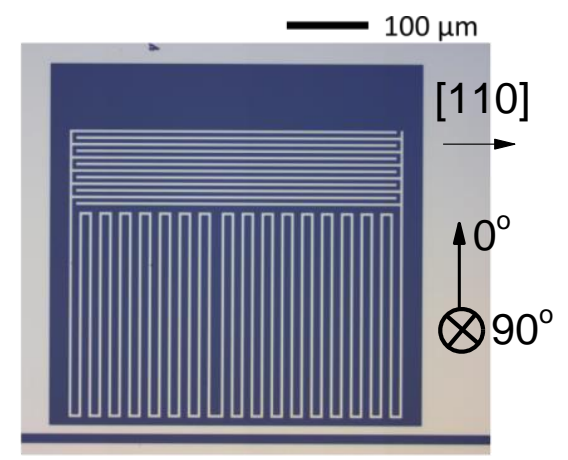

(c)

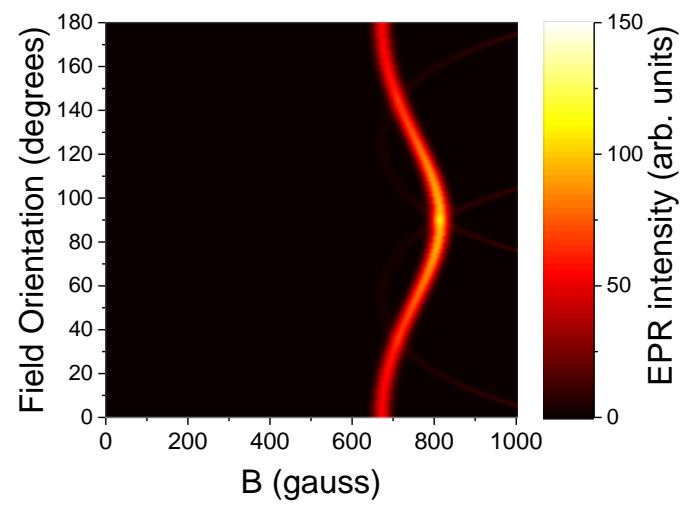

(b)

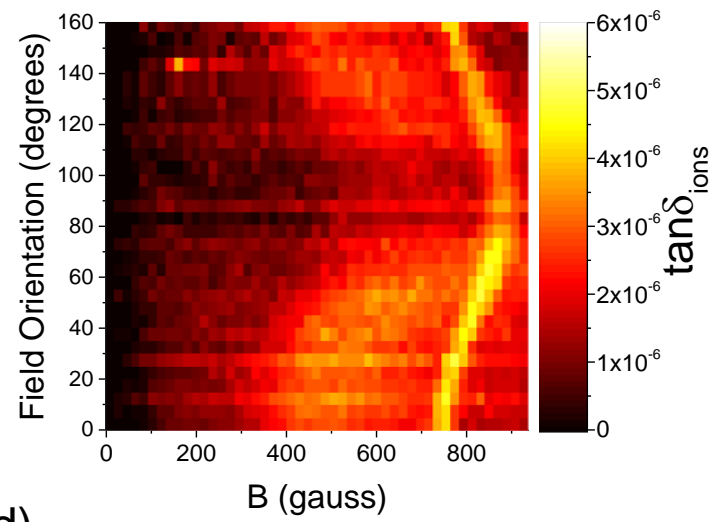

(d)

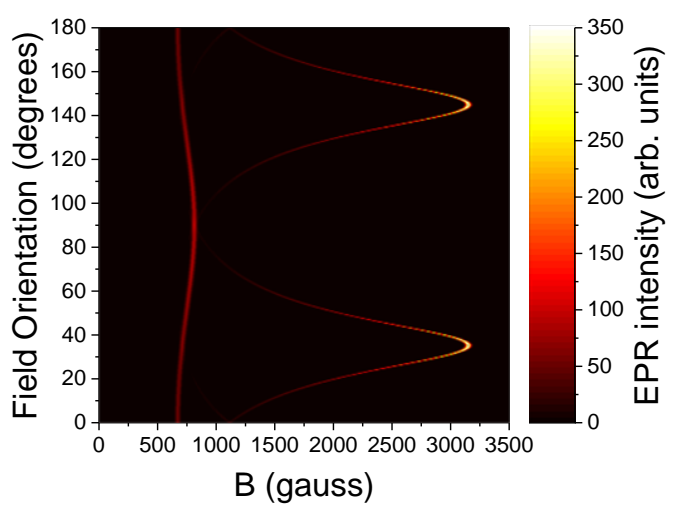

FIG 3 a) Image of the SC resonator that was coupled to the $10^{17} \mathrm{~cm}^{-3}$ Er sample. The [110] direction of the sample which was placed on top of and covered the resonator is shown, along with the $0^{\circ}$ and $90^{\circ}$ directions of the magnetic field. b) Angular dependent micro-resonator EPR measurement at $20 \mathrm{mK}$. c) simulated angular dependent EPR spectrum using EASYSPIN numerical modelling for the trigonal OEr-2' centre identified by Carey et al. [21] with $\mathrm{g}_{\|}=0.69$ and $\mathrm{g}_{\perp}=3.24$. d) Simulated angular dependent EPR extended to higher $\mathrm{B}_{0}$ to show the positions of the three expected EPR resonances with trigonal symmetry. The microwave frequency was $3.04 \mathrm{GHz}$ for all micro-resonator measurements and simulations.

The Q factor of a resonator coupled to an ensemble of spins can be modelled as a single mode harmonic oscillator according to

$$
Q_{t o t}=\frac{\Delta^{2}+\gamma^{2}}{2 g_{c o l}^{2} \gamma+\kappa\left(\Delta^{2}+\gamma^{2}\right)} \omega_{r}
$$

where $\Delta$ is the detuning from the spin resonance peak, $\gamma$ is the spin linewidth, $\kappa$ is the cavity linewidth $=2 \pi \omega_{\mathrm{r}} / \mathrm{Q}_{\text {tot }}=0.56 \mathrm{MHz}$ for the $0^{\circ}$ orientation and was independently measured away from the resonance for each $\mathrm{B}_{0}$ orientation, $\mathrm{Q}_{\text {tot }}$ is the total measured cavity $\mathrm{Q}$, and $\mathrm{g}_{\text {col }}$ is the collective coupling strength. Figure 4 shows the fitting of Eq. 5 to the $\mathrm{Q}_{\text {tot }}$ for the $0^{\circ}$ orientation which gives $\mathrm{g}_{\mathrm{col}} / 2 \pi=1$ $\mathrm{MHz}$ and $\gamma / 2 \pi=80 \mathrm{MHz}$. The average for all $\mathrm{B}_{0}$ orientations was $\mathrm{g}_{\mathrm{col}} / 2 \pi=1.1 \pm 0.3 \mathrm{MHz}$ and $\gamma / 2 \pi=$ 
$85 \pm 25 \mathrm{MHz}$. The coupling strength of an individual spin to the $\mathrm{SC}$ resonator is given by $\mathrm{g}_{\mathrm{i}}=\mathrm{g}_{\text {col }} / \sqrt{\mathrm{N}}$, where $\mathrm{N}$ is the number of spins coupled to the resonator; using the number of Er ions above the inductive element $\left(\right.$ Er dose $\times$ inductive element area $\left.=3.7 \times 10^{10}\right)$ gives a lower limit for $\mathrm{g}_{\mathrm{i}}$ of $\sim 6 \mathrm{~Hz}$, since the implantation yield is unknown for this EPR centre. This compares to $\mathrm{g}_{\mathrm{i}} \sim 70 \mathrm{~Hz}$ for $\mathrm{Er}$ implanted $\mathrm{Y}_{2} \mathrm{SiO}_{5}$ crystal [14]. We calculated the expected collective coupling strength $\mathrm{g}_{\text {col,calc }}$ by numerically simulating, then integrating over, the magnetic field and implanted ion distributions. Details of this simulation are given in the supplementary information [20], and further details can be found in refs.[32-36]. Our numerical simulation provided $\mathrm{g}_{\text {col,calc }} \sim 3 \mathrm{MHz}$, which is, to a first approximation, consistent with the measured $\mathrm{g}_{\mathrm{col}}$ and similar to what we have calculated previously for Gd implanted $\mathrm{Al}_{2} \mathrm{O}_{3}$ coupled to a micro-resonator [37]. We observed no change in $\omega_{\mathrm{r}}$ as $\mathrm{B}_{0}$ was swept through the Er spin resonance, indicating the system is operating in the weak coupling regime. The signal to noise ratio (SNR) was calculated from $S N R=\left(A_{\text {signal }} / A_{\text {noise }}\right)^{2}$, where $A_{\text {signal }}$ and $A_{\text {noise }}$ are the signal and noise amplitudes, respectively. For the microresonator EPR measurement shown in Figure 4 the SNR was $140 \pm 30$, this compares a SNR of $5 \pm 2$ for the EPR spectrum of the same OEr-2' centre, with a similar sweep time. Our micro-resonator measurement represents the first reported coupling of a SC resonator to a rare earth ensemble implanted in $\mathrm{Si}$, and a significant improvement in SNR compared to standard EPR. 


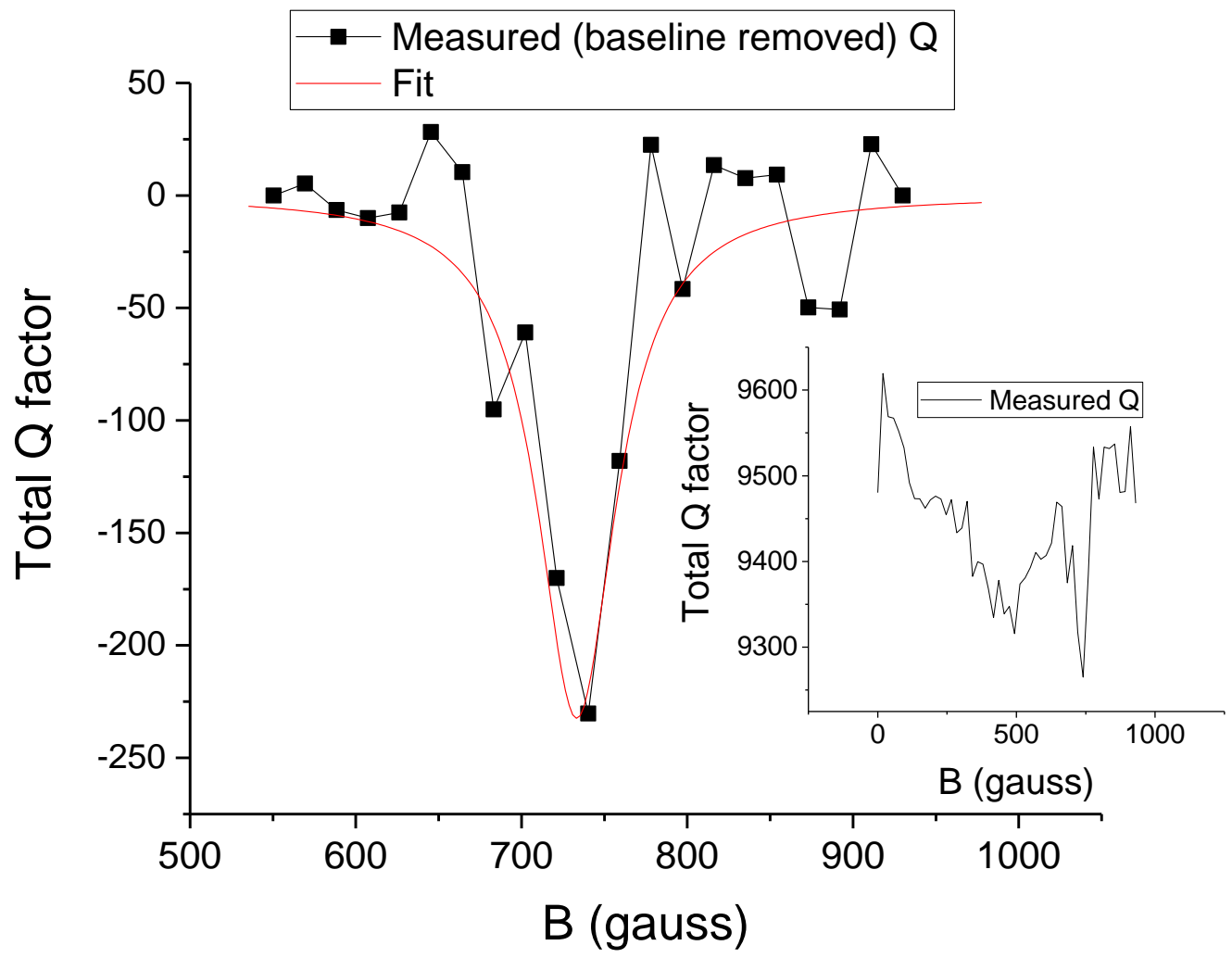

FIG 4. Measured Q factor with the baseline subtracted of the micro-resonator EPR measurement with a field orientation of $0^{\circ}$ with a fit using Eq. 5. Inset shows the total measured Q factor without baseline subtraction.

\section{CONCLUSIONS}

When Er concentration is reduced from $10^{19} \mathrm{~cm}^{-3}$ to $10^{17} \mathrm{~cm}^{-3}$, but the $\mathrm{O}$ concentration is kept at $10^{20}$ $\mathrm{cm}^{-3}$, no change in EPR linewidth was observed, indicating concentration effects are not important. The Si coordinated Er centre with cubic symmetry is no longer evident in PL measurements. Some small PL peak shifts of the O coordinated Er centre with orthorhombic symmetry were observed at $10^{17} \mathrm{~cm}^{-3}$ Er. Crystal field analysis of these PL peaks indicated that the orthorhombic Er centre has less tetragonal distortion, but greater orthorhombic distortion at $10^{17} \mathrm{~cm}^{-3} \mathrm{Er}$. Reducing Er concentration while keeping a relatively high $\mathrm{O}$ concentration may be an effective strategy to further reduce the numerous Er centres that are generated; however, detecting by standard EPR techniques presents a challenge to characterising at low concentrations, a solution could be to exploit SC resonator coupling. We observed the first coupling between a SC resonator and Er implanted Si with $\mathrm{g}_{\mathrm{col}}=1 \mathrm{MHz}$ and $\mathrm{g}_{\mathrm{i}}>6 \mathrm{~Hz}$, which provides a basis for characterisation of low concentration Er 
implanted Si and for future networks of hybrid quantum processors that exchange quantum information over the telecommunication network. Numerical simulation indicated an expected $\mathrm{g}_{\mathrm{col}}$ of $\sim 3 \mathrm{MHz}$. Out of six known Er-related EPR centres, only one trigonal centre coupled to the SC resonator at $20 \mathrm{mK}$. 


\section{References}

[1] A. J. Kenyon, Erbium in silicon, Semicond. Sci. Technol. 20, R65 (2005).

[2] M. A. Hughes, H. Li, N. Theodoropoulou, and J. D. Carey, Optically modulated magnetic resonance of erbium implanted silicon, Sci. Rep. 9, 19031 (2019).

[3] M. A. Hughes, M. A. Lourenço, J. D. Carey, B. Murdin, and K. P. Homewood, Crystal field analysis of Dy and Tm implanted silicon for photonic and quantum technologies, Opt. Express 22, 29292 (2014).

[4] M. A. Hughes, N. A. Panjwani, M. Urdampilleta, K. P. Homewood, B. Murdin, and J. D. Carey, Spin echo from erbium implanted silicon, Appl. Phys. Lett. 118, 194001 (2021).

[5] S. Welinski, P. J. Woodburn, N. Lauk, R. L. Cone, C. Simon, P. Goldner, and C. W. Thiel, Electron spin coherence in optically excited states of rare-earth ions for microwave to optical quantum transducers, Phys. Rev. Lett. 122, 247401 (2019).

[6] S. Bertaina, S. Gambarelli, A. Tkachuk, I. N. Kurkin, B. Malkin, A. Stepanov, and B. Barbara, Rare-earth solid-state qubits, Nat. Nanotechnol. 2, 39 (2007).

[7] P. Bushev, A. Feofanov, H. Rotzinger, I. Protopopov, J. Cole, C. Wilson, G. Fischer, A. Lukashenko, and A. Ustinov, Ultralow-power spectroscopy of a rare-earth spin ensemble using a superconducting resonator, Phys. Rev. B 84, 060501 (2011).

[8] S. Probst, A. Bienfait, P. Campagne-Ibarcq, J. Pla, B. Albanese, J. Da Silva Barbosa, T. Schenkel, D. Vion, D. Esteve, and K. Mølmer, Inductive-detection electron-spin resonance spectroscopy with 65 spins/Hz sensitivity, Appl. Phys. Lett. 111, 202604 (2017).

[9] Y. Kubo, F. Ong, P. Bertet, D. Vion, V. Jacques, D. Zheng, A. Dréau, J.-F. Roch, A. Auffèves, and F. Jelezko, Strong coupling of a spin ensemble to a superconducting resonator, Phys. Rev. Lett. 105, 140502 (2010).

[10] B. Julsgaard, C. Grezes, P. Bertet, and K. Mølmer, Quantum Memory for Microwave Photons in an Inhomogeneously Broadened Spin Ensemble, Phys. Rev. Lett. 110, 250503 (2013).

[11] G. Dold, C. W. Zollitsch, J. O'sullivan, S. Welinski, A. Ferrier, P. Goldner, S. de Graaf, T. Lindström, and J. J. Morton, High-cooperativity coupling of a rare-earth spin ensemble to a superconducting resonator using yttrium orthosilicate as a substrate, Physical Review Applied 11, 054082 (2019).

[12] S. Probst, H. Rotzinger, A. V. Ustinov, and P. A. Bushev, Microwave multimode memory with an erbium spin ensemble, Phys. Rev. B 92, 014421 (2015).

[13] J. H. Wesenberg, K. Mølmer, L. Rippe, and S. Kröll, Scalable designs for quantum computing with rare-earth-ion-doped crystals, Phys. Rev. A 75, 012304 (2007).

[14] S. Probst, N. Kukharchyk, H. Rotzinger, A. Tkalčec, S. Wünsch, A. D. Wieck, M. Siegel, A. V. Ustinov, and P. A. Bushev, Hybrid quantum circuit with implanted erbium ions, Appl. Phys. Lett. 105, 162404 (2014).

[15] J. J. Morton and P. Bertet, Storing quantum information in spins and high-sensitivity ESR, Journal of Magnetic Resonance 287, 128 (2018).

[16] L. A. Williamson, Y.-H. Chen, and J. J. Longdell, Magneto-optic modulator with unit quantum efficiency, Phys. Rev. Lett. 113, 203601 (2014).

[17] X. Fernandez-Gonzalvo, Y.-H. Chen, C. Yin, S. Rogge, and J. J. Longdell, Coherent frequency up-conversion of microwaves to the optical telecommunications band in an Er: YSO crystal, Phys. Rev. A 92, 062313 (2015).

[18] K. Groot-Berning, T. Kornher, G. Jacob, F. Stopp, S. T. Dawkins, R. Kolesov, J. Wrachtrup, K. Singer, and F. Schmidt-Kaler, Deterministic single-ion implantation of rare-earth ions for nanometerresolution color-center generation, Phys. Rev. Lett. 123, 106802 (2019).

[19] J. D. Carey, J. F. Donegan, R. C. Barklie, F. Priolo, G. Franzò, and S. Coffa, Electron paramagnetic resonance of erbium doped silicon, Appl. Phys. Lett. 69, 3854 (1996).

[20] See Supplemental Material at [URL will be inserted by publisher] for the simulated implant profile and numerical simulation of collective coupling strength. 
[21] J. D. Carey, R. C. Barklie, J. F. Donegan, F. Priolo, G. Franzo, and S. Coffa, Electron paramagnetic resonance and photoluminescence study of Er-impurity complexes in Si, Phys. Rev. B 59, 2773 (1999).

[22] H. Przybylinska, W. Jantsch, Y. Suprun-Belevitch, M. Stepikhova, L. Palmetshofer, G. Hendorfer, A. Kozanecki, R. J. Wilson, and B. J. Sealy, Optically active erbium centers in silicon, Phys. Rev. B 54, 2532 (1996).

[23] J. D. Carey, R. C. Barklie, J. F. Donegan, F. Priolo, G. Franzò, and S. Coffa, EPR study of erbium-impurity complexes in silicon, J. Lumines. 80, 297 (1998).

[24] J. D. Carey, Structure of multi-oxygen-related defects in erbium-implanted silicon, J. Phys.Condes. Matter 14, 8537 (2002).

[25] N. Q. Vinh, H. Przybylińska, Z. F. Krasil'nik, and T. Gregorkiewicz, Microscopic structure of Errelated optically active centers in crystalline silicon, Phys. Rev. Lett. 90, 066401 (2003).

[26] S. Laachir, M. Moussetad, and R. Adhiri, Crystal-Field energy levels of trivalent Erbium ion in cubic symmetry, Zeitschrift für Naturforschung A 66, 457 (2011).

[27] S. Probst, Hybrid quantum system based on rare earth doped crystals, Karlsruher Instituts für Technologie, 2015.

[28] W. Carnall, G. Goodman, K. Rajnak, and R. Rana, A systematic analysis of the spectra of the lanthanides doped into single crystal $\mathrm{LaF}_{3}$, The Journal of Chemical Physics 90, 3443 (1989).

[29] A. Abragam and B. Bleaney, Electron Paramagnetic Resonance of Transition Ions 1970).

[30] K. R. Lea, M. J. M. Leask, and W. P. Wolf, The raising of angular momentum degeneracy of $f-$ Electron terms by cubic crystal fields, J. Phys. Chem. Solids 23, 1381 (1962).

[31] P. Pal, T. Penhouet, V. D'Anna, and H. Hagemann, Effect of pressure on the free ion and crystal field parameters of $\mathrm{Sm}^{2+}$ in BaFBr and SrFBr hosts, J. Lumines. 134, 678 (2013).

[32] I. Wisby, PhD Thesis, Royal Holloway, University of London, 2017.

[33] J. Burnett, T. Lindström, M. Oxborrow, Y. Harada, Y. Sekine, P. Meeson, and A. Y. Tzalenchuk, Slow noise processes in superconducting resonators, Phys. Rev. B 87, 140501 (2013).

[34] A. Megrant, C. Neill, R. Barends, B. Chiaro, Y. Chen, L. Feigl, J. Kelly, E. Lucero, M. Mariantoni, and P. J. O'Malley, Planar superconducting resonators with internal quality factors above one million, Appl. Phys. Lett. 100, 113510 (2012).

[35] J. Burnett, L. Faoro, I. Wisby, V. Gurtovoi, A. Chernykh, G. Mikhailov, V. Tulin, R. Shaikhaidarov, V. Antonov, and P. Meeson, Evidence for interacting two-level systems from the $1 / f$ noise of a superconducting resonator, Nat. Commun. 5, 1 (2014).

[36] A. A. B. Brojeny, Y. Mawatari, M. Benkraouda, and J. R. Clem, Magnetic fields and currents for two current-carrying parallel coplanar superconducting strips in a perpendicular magnetic field, Superconductor Science and Technology 15, 1454 (2002).

[37] I. Wisby, S. E. de Graaf, R. Gwilliam, A. Adamyan, S. E. Kubatkin, P. J. Meeson, A. Y. Tzalenchuk, and T. Lindström, Coupling of a locally implanted rare-earth ion ensemble to a superconducting micro-resonator, Appl. Phys. Lett. 105, 102601 (2014).

\section{Acknowledgements}

This work was supported by the UK EPSRC grants EP/R011885/1 and EP/H026622/1. We would like to thank Prof. John Morton for helpful discussions.

\section{Author Contributions}


The concept was developed by MAH. Experimental work was performed by MAH, NAP, MU, NT and IW with input from JDC and TL. The manuscript was written by MAH with editorial input from JDC, $\mathrm{KPH}$ and $\mathrm{BM}$. All authors contributed to analyzing the results and commented on the paper.

\section{Data availability}

The datasets generated during the current study are available in the Mendeley Data repository at http://dx.doi.org/10.17632/stpfvbp8yc.1 